\title{
Una visión histórica de las drogas de abuso desde la perspectiva criminológica (Parte I)
}

\author{
An historical vision of drugs of abuse from the criminological \\ perspective (Part I)
}

\section{Resumen}

La relación entre el consumo de drogas (incluyendo el fenómeno de las drogodependencias) y la criminología es sumamente estrecha, aunque aún no se ha podido establecer un nexo causal definitivo entre ambas. En el presente trabajo se revisa la historia de las drogodependencias y del uso de drogas y de la normativa legal para combatir estos problemas, iniciada en EE.UU. (Pure Food and Drug Act, 1906) y que culminaría, a nivel internacional, con la Convención Única sobre Estupefacientes de Viena (1988). En la segunda parte de este trabajo se analizará el vínculo entre el uso/abuso de drogas y la delictogénesis.

Palabras clave: Drogas de abuso. Criminología. Delictogénesis. Historia.

\section{Abstract}

The relation between drug use (including drug addiction) and criminology is extremely narrow, although it has not yet been possible to establish a definitive causal nexus between both. In the present paper, we review the history of drug dependence and drug use and the legal norms to fight against these problems. It was initiated in the USA Pure Food and Drug Act (1906) and was culminated at an international level, with the Single Convention on Narcotic Drugs of Vienna (1988). In the second part of this paper, we will analyse the link between the drug use/abuse and the crime genesis.

Key words: Drugs of abuse. Criminology. Crime genesis. History.

\section{Introducción}

El complejo vínculo del ser humano con las sustancias psicotrópicas hay que enmarcarlo, no solamente en la vertiente sociosanitaria o jurídico-legal, sino en el más amplio círculo antropológico de la cultura. De hecho, las drogas se han empleado, por la mayor parte de las civilizaciones, como herramientas básicas para complementar sus ritos. Baste recordar el uso del fruto del guaraná por los indios brasileños, el hongo Amanita muscaria por los chamanes siberianos, el peyote por los indios huicholes, o el hachís por los hindúes ${ }^{1}$.

Sin embargo, este tipo de sustancias también han sido utilizadas desde épocas remotas como herramientas de placer o evasión, en una especie de antesala del actual fenómeno de la drogodependencia, aunque, desde la perspectiva histórica, no parece plausible afirmar que este fenómeno haya acompañado al ser humano desde sus orígenes. Salvo alguna excepción alarmante, como la epidemia de consumo de opio en China durante el siglo XIX, el problema de las drogas de abuso (y su conexión con el desarrollo de conductas delictivas) fue incrementándose paulatinamente en el mundo occidental durante el siglo $X X$, alcanzando su punto álgido en fechas relativamente recientes, de manos de la generación de la Guerra del Vietnam y del movimiento contracultural de Mayo del 68. Esta situación fue entendida por Naciones Unidas como un problema de Salud Pública, creándose un Programa de Fiscalización de

\author{
F. López-Muñoz ${ }^{1,2}$ \\ E. González ${ }^{3}$ \\ MD. Serrano ${ }^{4}$ \\ R. Antequera ${ }^{5}$ \\ C. Alamo ${ }^{1}$
}

${ }^{1}$ Departamento de Farmacología,

Facultad de Medicina, Universidad de Alcalá, Madrid.

${ }^{2}$ Facultad de Ciencias de la Salud, Universidad Camilo José Cela, Madrid.

${ }^{3}$ Equipo de Policía Judicial de la Guardia Civil, Valdepeñas,

Ciudad Real.

${ }^{4}$ Departamento de Derecho Penal y Criminología, Facultad de Derecho, UNED, Madrid.

5Instituto de Medicina Legal, Ciudad Real.

Correspondencia: Francisco López-Muñoz. E-mail: francisco.lopez.munoz@ gmail.com

Fecha de recepción: 4.NOV.2010

Fecha de aceptación: 15.FEB.2011 
Estupefacientes (PNUFID) y una Junta Internacional del mismo nombre (JIFE) para su control y erradicación.

En la actualidad, y aunque no existen conclusiones universalmente válidas, de lo que no cabe duda es que existe un estrecho vínculo entre la capacidad para cometer diferentes delitos (delictogénesis) y el entorno del sujeto consumidor y/o dependiente, lo que hace del consumo/dependencia de drogas un factor criminógeno de primer orden en nuestros días.

\section{Evolución histórica del fenómeno de las drogodependencias, del consumo de drogas y de su regulación legal}

\section{Opiáceos}

El opio (del griego opós = jugo [de la adormidera]), alcaloide extraído de la amapola, Papaver somniferum L., es una de las drogas más antiguas empleadas por el hombre. De hecho, su uso en varias culturas de Asia Menor, particularmente la asiria, se remonta a unos 4.000 años a.C., según citan diversas fuentes arqueológicas (su ideograma para la adormidera, hul-gil (planta del gozo), viene recogido en las famosas tablillas de arcilla de Uruk). El empleo terapéutico del opio como herramienta analgésica, antitusígena, hipnótica, sedante y tranquilizante, entre otras, ha sido una constante de la historia de la medicina ${ }^{2}$. Asimismo, el opio también ha desempeñado un trascendente papel histórico, desde el punto de vista político, comercial y sociológico.

La relación entre los opiáceos y la criminalidad se fundamenta básicamente en el fenómeno del narcotráfico, como posteriormente se comentará. No obstante, también existe un aspecto que podría ser calificado como "criminalidad a gran escala", que son los conflictos suprapersonales, muchas veces de carácter bélico, detrás de los cuales existen auténticos intereses de tipo económico, político o de cualquier otra naturaleza. Un ejemplo paradigmático lo constituyen las Ilamadas "Guerras del Opio", que tuvieron lugar durante el siglo $\mathrm{XIX}^{3}$. El uso del opio en China con fines medicinales y psicológicos tenía una larga historia, pero su consumo se hallaba limitado, pues la producción nacional era, en su conjunto, modesta. Sin embargo, a partir de 1773, Inglaterra inició un proceso de exportación masiva de opio hacia China, a través de la todopoderosa British East India Company, con objeto de equilibrar la balanza comercial con este país. La crisis económica generada, junto a la creación de un fenómeno de "drogodependencia de masas", impulsaron al gobierno chino a enfrentarse militarmente a Inglaterra, en las denominadas "Guerras del Opio" (1839-1842 y 1856-1860) (Figura 1a), que terminaron con la firma de los tratados de Nanking (1842) y de Pekín (1860), donde, concesiones territoriales aparte, como el caso de Hong Kong, China se convirtió en un inmenso mercado, con unos cien millones de potenciales consumidores de opio, cuyos pagos constituyeron una importante base económica del imperio británico ${ }^{4-6}$.

Otra consecuencia de esta generalización del consumo de opio (básicamente fumado) fue un espectacular incremento del cultivo de adormidera y de la producción de opio en China, donde, en 1906, se produjeron unas 30.000 toneladas de esta sustancia. Por otro lado, los viajeros occidentales instalados en China, así como los inmigrantes procedentes de Oriente, también introdujeron en Occidente la moda del consumo del opio fumado. En esa misma época, en Europa, la relativa facilidad para obtener el opio, integrado en la composición de distintos preparados terapéuticos, como elixires, anodinos o láudanos ${ }^{3}$, favoreció igualmente su abuso. El uso recreativo del opio también se vio favorecido por el negativo ejemplo de su consumo por parte de intelectuales reconocidos, como Sir Walter Scott, Samuel Taylor Coleridge, Thomas de Quincey, Honoré de Balzac, Edgar Allan Poe, Théophile Gautier, Charles Dickens o Charles Baudelaire. El aumento del número de adictos, junto a la presión ejercida por distintos movimientos sociales, dieron lugar a las primeras medidas restrictivas por parte de la administración norteamericana, de forma que en 1890 se estableció un impuesto específico sobre la importación de opio, y en 1899 se prohibió su consumo fumado, sobre todo en locales concretos ("fumaderos de opio").

El siglo XIX también supone el inicio de la historia de las drogas sintéticas. En 1803, el farmacéutico alemán Friedrich Wilhelm Sertürner inició sus primeros experimentos químicos, consiguiendo extraer un ácido orgánico a partir del opio, al que llamó ácido mecónico (del griego mekon = amapola), del cual obtuvo una sustancia dotada de efectos narcóticos, a la que denominó morphium (morfina), en honor de Morfeo (hijo del dios del sueño y dios del ensueño) $)^{7,8}$. La morfina fue comercializada en 1827 por la compañía E. Merck (Darmstadt, Alemania), aunque su uso no comenzó a difundirse ampliamente hasta mitad del siglo XIX, cuando Charles Gabriel Pravaz y Alexander Wood descubrieron, respectivamente, la jeringa y la aguja hipodérmica ${ }^{9-11}$. Precisamente, estos avances médicos ocasionaron otro grave problema de adicción en Estados Unidos, tras 
la finalización de la Guerra de Secesión Americana (1861-1865), pues durante este conflicto se les proporcionó a los soldados una jeringa hipodérmica y una dotación de morfina para administración parenteral, que podía autoadministrarse como analgésico en casos de lesiones o heridas de guerra. Al final del conflicto, la sociedad americana tuvo que enfrentarse a una nueva epidemia, conocida como "enfermedad del soldado" o el "mal militar" (Figura 1 b), que no era otra cosa que un síndrome de dependencia a este agente opiáceo, que había sido suministrado a los combatientes de forma absolutamente incontro$\operatorname{lada}^{10}$. Se estima que, al final de la Guerra de Secesión, había unos 45.000 soldados dependientes de la morfina ${ }^{1}$.

Dado el elevado número de adictos a la morfina, se impuso la necesidad de encontrar una nueva sustancia con una potencia analgésica semejante, pero sin problemas de dependencia. Así, el grupo de Heinrich Dreser, de la compañía alemana F. Bayer \& Co. (Elberfeld, Alemania), sintetizó en 1874 la diacetilmorfina. Este derivado de la morfina, tres veces más potente, fue finalmente comercializado en 1899 para el tratamiento de enfermedades respiratorias, con el nombre comercial de Heroína, acepción derivada de la palabra germana "heroish", que viene a significar remedio heroico o poderoso ${ }^{2}$. La heroína adquirió un rápido éxito comercial, siendo utilizada ampliamente en todo el mundo. Sin embargo, su potente capacidad adictiva no fue advertida hasta 1913, momento en que la compañía fabricante detuvo su producción.

El gran aumento del número de adictos al opio y a la morfina en Estados Unidos generó auténticos movimientos sociales durante el periodo de entresiglos, que presionaron a la Administración para que legislara medidas restrictivas del comercio de estas sustancias ${ }^{3}$. Como consecuencia de ello, en 1906, el gobierno de Theodore Roosevelt promulgó la Pure Food and Drug Act, que perseguía la adulteración de todos los preparados farmacéuticos, entre ellos los que contenían opiáceos. En 1909 tuvo lugar también, promovida por Estados Unidos, la primera iniciativa de alcance internacional para el control de los opiáceos, la Conferencia de Shangai, en la que representantes de trece países se hicieron presentes, pero se mostraron reacios ante la propuesta norteamericana de emprender una cruzada contra el uso recreativo de los opiáceos. Tres años después, en 1912, se firmó el primer instrumento internacional sobre esta materia, el Convenio contra el Opio de la Haya, que pretendía disminuir la producción y exportación de dicha sustancia. Sin embargo, este Convenio tuvo un valor más bien simbólico, dado que careció de fuerza vinculante para las partes.

La primera ley que restringió, de forma taxativa, el consumo de opiáceos fue la Harrison Narcotic Tax Act, de 1914, que imponía controles estrictos sobre la importación, la manufactura y distribución, prohibiendo su venta y dispensación, excepto por médicos y farmacias inscritos en un registro federal norteamericano ${ }^{1}$. Finalmente, en 1924 se ilegalizó la heroína en Estados Unidos, lo que generó un creciente tráfico clandestino de materias primas para la elaboración de este opiáceo. Éstas procedían de países del sudeste asiático (el denominado "triángulo de oro"), pasaban por Marsella (la denominada "French connection") y acababan en Norteamérica, donde grupos de inmigrantes italianos ya habían generado toda una red clandestina de distribución, aprovechando la infraestructura establecida para la distribución ilegal de alcohol durante la denominada "Ley Seca". Estos grupos fueron incrementando su papel

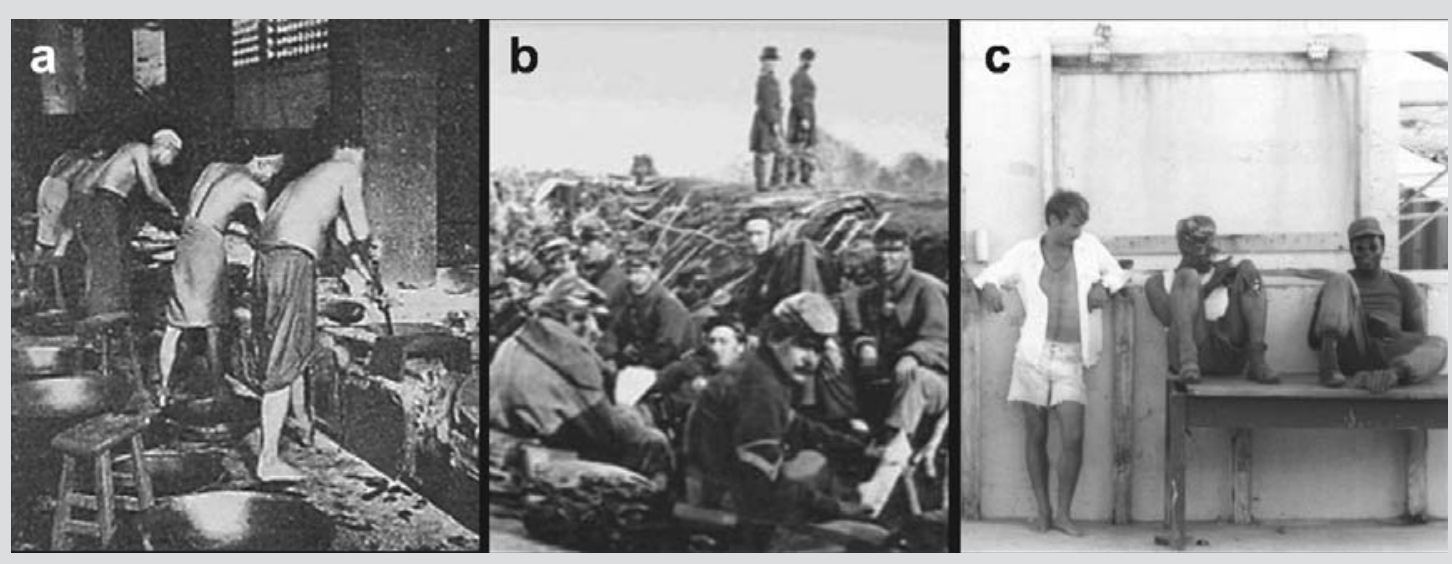

Figura 1a.

Las Guerras del Opio entre el Imperio Británico y China. Fábrica de procesamiento del opio en China a finales del sig/o XIX; Figura $1 b$.

La morfina y la Guerra Civil Americana. Muchos veteranos de guerra acabaron siendo adictos a la morfina, ampliamente utilizada, sin control, como agente analgésico;

Figura 1c. La heroína y la Guerra de Vietnam. Un grupo de soldados adictos a la heroína en la puerta de una barraca de una base americana. 
en el narcotráfico durante las décadas siguientes, aunque las leyes contra el tráfico y el consumo de drogas se endurecieron notoriamente en la década de 1950. A título de ejemplo, la ley para el Control de Narcóticos, de 1956, llegaba incluso a establecer la pena de muerte por la venta de heroína a menores de edad.

Paralelamente, fueron surgiendo las primeras leyes internacionales de lucha contra los opiáceos, iniciadas con la Convención Única sobre Estupefacientes de Nueva York, en 1961, y complementadas con las Convenciones de Viena de 1971 y 1988, éstas sí, con fuerza vinculante para todas las partes, y que constituyen el marco de referencia de todas las legislaciones nacionales (Tabla 1). Entre las medidas adoptadas, en 1967 se creó la JIFE, cuyo objetivo principal era vigilar el cumplimiento de los tratados internacionales sobre este tipo de sustancias narcóticas. Todas estas normas restrictivas han contribuido al diseño de una política criminal en materia de drogas de carácter prohibicionista, que, sin duda, ha fomentado la generación de un mercado negro y tráfico ilegal que, como se describirá posteriormente, ha generado una serie de fenómenos de delincuencia asociada, de evolución constante hasta nuestros días ${ }^{3}$.

Uno de los puntos de inflexión en la historia de la heroína hay que buscarlo en la Guerra de Vietnam (1961-1975). A principios de la década de 1970, el consumo de drogas en algunas unidades del ejército americano en Vietnam alcanzó proporciones casi epidémicas (Figura 1c) y muchos de los ex-combatientes adictos acabaron, tras el regreso a su país, en el mundo de la delincuencia. Simultáneamente, con el auge de la cultura "underground" y el movimiento hippie se abogó por el consumo de todo tipo de drogas, expandiéndose el consumo de heroína por todo el mundo. En el ámbito de la Unión Europea, el problema del consumo de heroína fue en aumento hasta el año 1992, fecha en la que parece haberse estabilizado. A título de ejemplo, la Tabla 2 muestra la evolución del consumo de heroína en España, que apenas ha variado en la última década ${ }^{12}$. En cualquier caso, se ha estimado que en la Unión Europea y Noruega existirían 1,3-1,7 millones de consumidores problemáticos de opiáceos ${ }^{13}$ y que la heroína ha generado más muertes en los últimos 50 años, por vía directa o indirecta, que cualquier otra sustancia química conocida ${ }^{14,15}$.

\section{Cocaína}

El consumo de la hoja de la coca (Erythroxylon coca, L.) también viene realizándose desde épocas muy remotas por parte de los indígenas sudamericanos, habiéndose encontrado vasijas e instrumentos para su masticación en las costas chilena y peruana con una antigüedad de unos 4.000 años. La palabra coca procede de "khoca", que significa literalmente "árbol" o "planta". Desde el imperio inca (12001553 d.C.), la utilización masticada de las hojas de coca era parte de los rituales religiosos y sociales. Del mismo modo, se utilizaron con fines diagnósticos por parte de los sacerdotes incas, quienes diagnosticaban la causa de la enfermedad interpretando la disposición de las hojas arrojadas al suelo o el lugar al que se dirigía el jugo de coca escupido sobre la mano ${ }^{16}$. En 1507, Americo Vespucio ya refiere el hábito de los nativos de masticar hojas de coca con polvo de cal para fortalecerse. Asimismo, en 1532, Francisco Pizarro, al conquistar el Perú, observó que los indios peruanos masticaban hojas de coca y que de esta manera aumentaba su capacidad de trabajo y estaban en condiciones de hacer tareas que requerían un gran esfuerzo físico ${ }^{1}$. También Ilamó la atención sobre los síntomas de la intoxicación en los llamados "coqueros": "caminar vacilante e incierto, con una carne flácida, amarillenta, de ojos hundidos y sin brillo". La planta de la coca fue descrita por primera vez por el religioso dominico español fray Tomás Ortiz, en 1499, y de manos de los conquistadores españoles llegó a Europa, con el nombre de "yerba del Paraguay"16. Durante la Ilustración, uno de los mayores valedores de la coca fue Hermann Boerhaave. En 1708 publicó sus Instituciones Médicas, que contenían una revisión de las hierbas con valor medicinal, y entre ellas se encontraba la coca. Boerhaave inició un importante cambio en la actitud pública hacia esta planta, relatando diferentes utilidades médicas ${ }^{17}$. En esta época se agudizó la larga controversia, que se prolongó durante el siglo siguiente, sobre los beneficios de la coca. La opinión médica también estaba dividida, pues mientras algunos participaban de la idea de Boerhaave de que se trataba del "elixir de la vida", otros la consideraban una engañosa piedra filosofal ${ }^{16-18}$.

Las dificultades para aislar su alcaloide influyeron para que no fuese considerada una planta con principios activos hasta la segunda mitad del siglo XIX. En 1858, el médico de la fragata austriaca Novara, doctor Karl Ritter von Scherzer, trajo a Europa unas hojas secas de coca. Scherzer entregó las hojas al químico alemán Friedrich Wöhler, de Göttingen, para que las analizara y, finalmente, un discípulo suyo, Albert Niemann, aisló en 1859 el principio activo de estas hojas, al que denominó "cocaína", aunque, esencialmente, lo que hizo fue redescubrir la eritroxilina, pero mejorando la técnica. La estructura química de la cocaína no sería descrita hasta 1895 , gracias a los trabajos del químico alemán Richard Willstätter ${ }^{16-18}$. La cocaína se comercializó (en 1882 en Estados Unidos) como un fármaco eficaz para el tratamiento de las enfermedades nerviosas y los trastornos depresivos, además de como anestésico local 


\begin{tabular}{|c|c|c|}
\hline Año & Lugar & Tratado - Convenio \\
\hline 1796 & & $\begin{array}{l}\text { China fue el primer país que tomó conciencia sobre los peligros del opio, } \\
\text { decretando que su uso se castigara con la muerte. }\end{array}$ \\
\hline 1858 & & $\begin{array}{l}\text { El tratado de Tientsin impuesto a China por Francia, Inglaterra, Rusia y } \\
\text { los Estados Unidos condujo a la legislación del comercio del opio. }\end{array}$ \\
\hline 1875 & EE.UU. & $\begin{array}{l}\text { La primera ley antidrogas de Estados Unidos se decretó en } 1875 \text { contra } \\
\text { las "casas de opio", dictada en la ciudad de San Francisco. }\end{array}$ \\
\hline 1887 & EE.UU. & $\begin{array}{l}\text { Ley que prohibía que los ciudadanos chinos importaran opio a EE.UU. } \\
\text { para fumar. }\end{array}$ \\
\hline 1906 & EE.UU. & Pure Food and Drug Act. \\
\hline 1909 & Shangai & $\begin{array}{l}\text { Primera Comisión Internacional del Opio, celebrada en Shangai con } 13 \\
\text { representantes. }\end{array}$ \\
\hline 1912 & La Haya & Convenio Internacional del Opio. \\
\hline 1914 & EE.UU. & $\begin{array}{l}\text { Harrison Narcotic Tax Act. } \\
\text { Controles estrictos sobre la importación, la manufactura y la distribución } \\
\text { de opio y opiáceos. } \\
\text { Queda prohibida la venta de dosis sustanciales de opiáceos o cocaína, } \\
\text { excepto por fabricantes, médicos y farmacias. }\end{array}$ \\
\hline 1924 & EE.UU. & Heroin Act. \\
\hline 1925 & Ginebra & II Convenio Internacional del Opio. \\
\hline 1929 & EE.UU. & $\begin{array}{l}\text { "Ley seca" (18 a enmienda a la Constitución de los Estados Unidos - } \\
\text { Volstead Act-): prohibición para la fabricación, transporte o venta de } \\
\text { bebidas alcohólicas. }\end{array}$ \\
\hline 1931 & Ginebra & $\begin{array}{l}\text { Convenio para limitar la fabricación y reglamentar la distribución de } \\
\text { estupefacientes. }\end{array}$ \\
\hline 1936 & Ginebra & Convenio para la represión del tráfico ilícito de las drogas peligrosas. \\
\hline 1937 & EE.UU. & $\begin{array}{l}\text { Marihuana Tax Act: Prohibición del cultivo, distribución y venta de } \\
\text { marihuana. }\end{array}$ \\
\hline 1942 & EE.UU. & $\begin{array}{l}\text { Opium Poppy Control Act: Reglamentación del cultivo de la amapola y el } \\
\text { comercio del opio. }\end{array}$ \\
\hline 1946 & Nueva York & $\begin{array}{l}\text { Protocolo enmendando los acuerdos, convenciones y protocolos sobre } \\
\text { estupefacientes concertados. }\end{array}$ \\
\hline 1956 & EE.UU. & Narcotic Control Act: endurecimiento de las penas por narcotráfico. \\
\hline 1961 & Nueva York & $\begin{array}{l}\text { Convención Única sobre Estupefacientes. } \\
\text { Regulación de las drogas provenientes de plantas como la amapola, el } \\
\text { cáñamo y la coca. }\end{array}$ \\
\hline 1967 & Viena & $\begin{array}{l}\text { Creación de la Junta Internacional de Fiscalización de Estupefacientes } \\
\text { (JIFE), para vigilar el cumplimiento de los tratados internacionales sobre } \\
\text { estupefacientes. }\end{array}$ \\
\hline 1971 & Viena & $\begin{array}{l}\text { Convenio sobre Sustancias Psicotrópicas. } \\
\text { Inclusión de nuevas drogas en las listas de sustancias prohibidas } \\
\text { sometidas a fiscalización. }\end{array}$ \\
\hline 1972 & Ginebra & $\begin{array}{l}\text { Protocolo de modificación de la Convención Única sobre Estupefacientes } \\
\text { de } 1961 .\end{array}$ \\
\hline 1973 & EE.UU. & $\begin{array}{l}\text { Drug Enforcement Administration (DEA): Agencia de lucha contra el } \\
\text { narcotráfico. }\end{array}$ \\
\hline 1988 & Viena & $\begin{array}{l}\text { Convención de las Naciones Unidas contra el tráfico ilícito de } \\
\text { estupefacientes y sustancias psicotrópicas. }\end{array}$ \\
\hline 1991 & U.E. & $\begin{array}{l}\text { Directiva 91/308/CEE del Consejo, de } 10 \text { de junio de } 1991 \text {, relativa a la } \\
\text { prevención de la utilización del sistema financiero para el blanqueo de } \\
\text { capitales. }\end{array}$ \\
\hline 2005 & U.E. & $\begin{array}{l}\text { Reglamento (CEE) no } 111 / 2005 \text { del Consejo, de } 22 \text { de diciembre de } \\
2004 \text {, sobre precursores químicos para la producción de drogas. }\end{array}$ \\
\hline
\end{tabular}

Tabla 1.

Cronograma-resumen de la legislación y los tratados internacionales más relevantes en materia de consumo de drogas y de lucha contra el tráfico de drogas. 
en intervenciones oculares y para el tratamiento del dolor odontológico en la infancia ${ }^{18}$. La popularidad de la cocaína creció rápidamente y entró a formar parte de la composición de numerosos "elixires milagrosos" que se vendían, en la época del cambio de siglo, por sus propiedades energizantes y vigorizantes. El más famoso de todos fue el desarrollado por el químico y farmacéutico corso Angelo Mariani, que elaboró un vino con extractos de hojas de coca patentado como "Vino Mariani". Mariani fundó, en 1863 , la primera gran industria basada en la coca. Tal fue el éxito de este vino que Mariani recibió una condecoración del Papa León XIII por sus méritos en pro de la Humanidad. En Estados Unidos, donde el alcohol no gozaba de buena reputación en un amplio sector de la sociedad, John Styth Pemberton formuló en 1885 un sucedáneo del Vino Mariani libre de alcohol, al que Ilamó "French Wine Coca". Este tónico y estimulante nervioso fue reformulado al año siguiente bajo el nombre de "Coca-Cola". La compañía Coca-Cola fue fundada en 1892 y durante la década de los 90 anunciaba su producto como remedio para el dolor de cabeza y como estimulante, además de como una agradable bebida: "bebida medicinal intelectual y para el temperamento". En 1903, la compañía Coca-Cola eliminó la cocaína de su bebida, sustituyéndola por cafeína, aunque mantuvo las hojas de coca descocainizadas como aromatizante. En 1909, estaban comercializadas en Estados Unidos unas 69 bebidas que contenían cocaína como ingrediente.

De forma simultánea al aumento de su uso, aparecieron los primeros casos descritos de dependencia, así como las primeras notificaciones de reacciones psicóticas, crisis convulsivas e incluso casos de muerte súbita. Igual que sucedió con los opiáceos, la Pure Food and Drug Act, de 1906, puso las primeras restricciones a su manufacturación y, en 1907, el
Estado de Nueva York limitó la distribución de la cocaína exclusivamente bajo control médico, lo que hizo aparecer un importante mercado negro ${ }^{19}$. En 1914, la cocaína fue ilegalizada completamente en Estados Unidos, en aplicación de la Harrison Narcotic Act, salvo en su uso terapéutico y de investigación por parte de médicos y laboratorios, que debían inscribirse en un registro especial. Ese mismo año, con la propuesta de "controlar la preparación de opio, morfina y cocaína", varios países adoptaron los planteamientos de la Convención de la Haya de 1912, ratificados en 1919 en el Tratado de Versalles (Tabla 1 ).

Sin embargo, la popularidad de la cocaína como "droga recreativa" fue aumentando durante la década de 1920, alcanzando un cierto prestigio entre aristócratas, burgueses y bohemios; pero con el final de los frívolos años veinte, el patrón de consumo pasó al mundo de los homosexuales, prostitutas y gente del espectáculo y del cabaret ${ }^{19}$. El patrón de consumo de la cocaína volvió a invertirse durante la década de 1970, convirtiéndose en la droga de las estrellas de rock, los corredores de bolsa, etc. Pasó a ser la "droga de los ricos" y de la élite, la "droga del bienestar", cuyo consumo se asociaba a un cierto estatus social privilegiado. La Tabla 2 muestra como el consumo de cocaína no ha parado de crecer durante la última década en España, el país de la Unión Europea donde la prevalencia del consumo de esta sustancia es mayor (un $8 \%$ de la población la ha probado alguna vez en la vida) ${ }^{12}$. Por su parte, en el conjunto de la Unión Europea, unos 14 millones de adultos han consumido cocaína a lo largo de la vida $\left(4,1 \%\right.$ de la población europea adulta ${ }^{20}$.

El incremento paulatino del consumo mundial de cocaína durante las últimas décadas se ha acompañado de un aumento del contrabando y del precio.

Tabla 2.

Evolución de la prevalencia de consumo de drogas en la población española de 15-64 años (\%).

\begin{tabular}{|c|c|c|c|c|c|c|c|c|c|c|c|c|c|c|}
\hline & \multicolumn{2}{|c|}{1995} & \multicolumn{2}{|c|}{1997} & \multicolumn{2}{|c|}{1999} & \multicolumn{2}{|c|}{2001} & \multicolumn{2}{|c|}{2003} & \multicolumn{2}{|c|}{2005} & \multicolumn{2}{|c|}{$2007 / 08$} \\
\hline & $\mathrm{A}$ & $\mathrm{B}$ & A & $\mathrm{B}$ & $\mathrm{A}$ & B & $\mathrm{A}$ & $B$ & $\mathrm{~A}$ & B & $\mathrm{A}$ & $\mathrm{B}$ & $\mathrm{A}$ & $\mathrm{B}$ \\
\hline Cannabis & 14,5 & 7,5 & 22,9 & 7,7 & 19,6 & 7,0 & 23,8 & 9,2 & 29,0 & 11,34 & 28,6 & 11,2 & 27,3 & 10,1 \\
\hline Extasis & 2,0 & 1,3 & 2,5 & 0,9 & 2,4 & 0,8 & 4,0 & 1,8 & 4,6 & 1,4 & 4,4 & 1,2 & 4,3 & 1,1 \\
\hline Alucinógenos & 2,1 & 0,8 & 2,9 & 0,9 & 1,9 & 0,6 & 2,8 & 0,7 & 3,0 & 0,6 & 3,4 & 0,7 & 3,8 & 0,6 \\
\hline Anfetaminas & 2,3 & 1,0 & 2,7 & 0,9 & 2,2 & 0,7 & 2,9 & 1,1 & 3,2 & 0,8 & 3,4 & 1,0 & 3,8 & 0,9 \\
\hline Cocaina (polva) & 3,4 & 1,8 & 3,4 & 1,6 & 3,1 & 1,6 & 4,8 & 2,5 & 5,9 & 2,7 & 7,0 & 3,0 & 8,0 & 3,0 \\
\hline Heroína & 0,8 & 0,5 & 0,6 & 0,2 & 0,5 & 0,1 & 0,6 & 0,1 & 0,9 & 0,1 & 0,7 & 0,1 & 0,8 & 0,1 \\
\hline Inhalables & 0,7 & 0,1 & 0,8 & 0,2 & 0,6 & 0,1 & 0,8 & 0,1 & 1 & 0,1 & 0,8 & 0,1 & 1,1 & 0,1 \\
\hline
\end{tabular}

Dotos del Observatorio Esponiol sobre Drogas, $2008^{22}$. 
Sin embargo, las consecuencias sociales para los países productores han sido dramáticas, con un cambio de cultivos de los campesinos sudamericanos, hacia estos cultivos más "beneficiosos". Del mismo modo, fueron (y siguen siendo) dramáticos los efectos criminológicos, con un aumento exponencial del narcotráfico, la aparición de los sangrientos "cárteles de la droga", etc. ${ }^{3}$. En los últimos años, el mercado de la cocaína ha continuado su crecimiento, debido a varios factores: el acoso que está sufriendo el mercado de la heroína; su gran disponibilidad y el descenso de su precio, al disminuir el coste del proceso de fabricación; el poseer la consideración de bajo peligro social y sanitario; su empleo, fundamentalmente por vía intranasal, evitando los riesgos de infecciones (HIV, fundamentalmente) asociadas a la administración parenteral. Además, se ha observado, sobre todo en Norteamérica, un incremento del consumo de fórmulas alternativas y mixtas, como el "speed ball", una mezcla de heroína y cocaína.

\section{Cannabis}

El cáñamo (Cannabis sativa) es otra de las plantas que ha acompañado al hombre desde épocas muy remotas, habiéndose empleado como sustancia de abuso bien su resina (hachís) o sus hojas (marihuana). Los cultivos más antiguos de la planta datan del año 3500 a.C. en el Himalaya, aunque su nombre parece provenir del término quannabu, con que la conocían los asirios ${ }^{21}$. Los primeros restos de su uso recreacional se han encontrado en la península indostánica, y han sido datados en siglo XV a.C. En esta cultura, el cannabis es mencionado en los primeros Vedas, sobre todo en el cuarto o Atharva Veda, como la bebida preferida del dios guerrero Indra. Los textos sánscritos de la India citan las Ilamadas "píldoras de la alegría", a base de cannabis y azúcar. En China, donde se empleaba en la industria textil, ya se cita este remedio en el Pen Tsao Ching, un tratado médico del siglo I atribuido al mítico emperador Shen Nung (siglo XXX a.C.), donde se recomendaba el uso del cannabis en casos de dolores reumáticos ${ }^{21}$, aunque tomado en exceso su consumo hacía que se viesen demonios y tomada durante mucho tiempo favorecía la comunicación con los espíritus y aligeraba el cuerpo. Por su parte, en la India, la medicina ayurvédica ya recogía la utilidad analgésica del cáñamo desde el siglo XV a.C., especialmente como antimigrañoso. El cannabis también se encontraba en el arsenal terapéutico de la medicina clásica griega y romana. Plutarco, escribiendo sobre los tracianos, unos 400 años después, refería la costumbre que tenían, tras la comida, de quemar las hojas de una planta parecida al orégano, aspirando sus vapores, lo que les producía un estado de embriaguez, cayendo finalmente en un profundo sueño. Este consumo también es citado por Cayo Plinio Segundo, al igual que por Dioscórides, en su Materia Medica, donde lo señala como útil en los dolores de cabeza y anafrodisiaco, siendo ésta la primera referencia al empleo clínico de la planta en un texto médico occidental ${ }^{22}$. Por su parte, Galeno describe la costumbre de usarlo como euforizante e hilarante en los banquetes, donde se tomaba en forma de pastel preparado con las semillas del mismo 23 .

El cannabis es una planta que se ha visto inmersa en numerosas leyendas, algunas de tintes claramente criminológicos, como la que la relaciona con los ismaelitas, pueblo shihita que se hizo depositario de los tratados alquímicos griegos sobre los efectos del cannabis. Así, cabe recordar la famosa secta de los hashashins, cuyo líder, Hasan-Ibn-al-Sabbah (el "Viejo de la Montaña"), hacía beber una cocción de cannabis a sus soldados como premio cada vez que iniciaban una batalla o tenían que cometer un "asesinato", casi siempre por encargo y previo pago ${ }^{24}$. Este hecho ha dado lugar a la teoría de que la palabra "assassins", como se conocía a sus seguidores, deriva de hashish ${ }^{3}$.

Aunque posiblemente los Cruzados trajeron esta sustancia a su retorno de Tierra Santa, la importación definitiva a Occidente de preparados psicotrópicos elaborados a partir del cáñamo fue efectuada por las tropas de Napoleón, tras su regreso de las campañas egipcias, alcanzando su consumo un gran éxito entre los intelectuales, como los integrantes del selecto "Club de los Hachichins" (Théophile Gautier, Charles Baudelaire, Eugène Delacroix, Gérard de Nerval, Gustave Flaubert, Alexandre Dumas), que, en sus reuniones en el elegante y decadente Hotel Lauzun, del barrio latino de París, tomaban el dawamesk, una mezcla de hachís, canela, clavo, pistacho, azúcar, zumo de naranja, mantequilla y cantáridas ${ }^{24}$. De sus experiencias con el hashish, Gautier publicó dos textos; el primero, en 1843, bajo el epígrafe Le Hashísh, y el segundo, en 1846, titulado Le Club des Hachichins, que apareció en la Revue des Deux Mondes. También Baudelaire publicó, dentro de su libro Los paraísos artificiales, un poema dedicado al mismo. En América, fueron los españoles los que introdujeron el cáñamo hacia 1547, adquiriendo gran relevancia en Norteamérica en años sucesivos; de hecho, en plena Guerra de Secesión, el mismo George Washington sembraba esta planta, siendo la segunda cosecha en importancia en Norteamérica, después del algodón. 
Aunque los primeros estudios del siglo XIX sobre el cannabis corresponden a Julien J. Virey, que buscó los principios activos de la planta, publicando sus resultados en 1803, los efectos psicotrópicos de esta sustancia fueron estudiados en profundidad por Jacques-Joseph Moreau de Tours, tanto como medio para conocer las alteraciones de conciencia que produce, por medio del autoconsumo, como desde la perspectiva terapéutica, donde finalmente quedó reducido a un mero uso como sedante. Sus resultados fueron publicados en 1845 en su celebérrimo libro Du haschich et de l'aliénation mentale - etudes psychologiques. Sin embargo, se considera que el introductor del cannabis en la medicina occidental fue médico irlandés William Brooke O'Shaughnessey, profesor del Colegio Médico de Calcuta, donde aprendió a utilizarlo. Este autor publicó, en 1839, el primero de una serie de artículos sobre el cannabis, On the preparations of the Indian hemp, or guhjah. Their effects on the animal system in health, and their utility in the treatment of tetanus and other convulsive diseases, donde elogia sus propiedades anticonvulsivantes. En 1842, de vuelta a Londres, entró en contacto con el farmacéutico Peter Squire, consiguiendo producir el primer extracto comercial de cannabis, conocido como el "Squire's Extract". El uso de los derivados de cannabis se extendió notablemente en las últimas décadas del siglo XIX, pudiéndose obtener en las farmacias de la época diferentes especialidades, como el Chlorodyne, un preparado del laboratorio Squibb \& Co. para los trastornos gástricos, que incluía también morfina, o las píldoras antineurálgicas de Brown Sequard. Finalmente, investigadores de la Universidad de Cambridge (Word, Spivey y Easterfield) obtuvieron en 1890 el primer extracto casi puro de la planta, al que Ilamaron cannabinol, y durante esa última década del siglo XIX acabaron obteniéndose otros alcaloides (cannabin, cannabindon, cannabine, cannabinon), algunos de los cuales se incorporaron a los diferentes preparados comerciales del momento. A finales del siglo XIX, el cannabis o hachís se encontraba presente ya en todas las farmacopeas occidentales y era ampliamente utilizado en la práctica médica para el tratamiento de neuralgias, reumatismos nerviosos, insomnio, crisis epilépticas, espasmos musculares, hemorragias uterinas, dismenorreas, etc. ${ }^{25}$. Sin embargo, su uso terapéutico declinó a partir de la década de 1930, tras su eliminación, en 1932, de la Farmacopea Británica ${ }^{26}$.

Durante la década de 1930 comenzaron las grandes migraciones de mexicanos hacia el sur de Estados Unidos, quienes exportaron a esas tierras la costumbre de consumir derivados psicoactivos del cáñamo (marijuana) con fines recreativos. El problema deri- vado de este consumo fue de tal importancia que, en 1930, se creó la Oficina Federal de Narcóticos, destinada únicamente al control del cultivo, venta y consumo de la planta ${ }^{1}$. De hecho, en 1937 se estimaba que había 100.000 adictos a la marihuana en Estados Unidos, principalmente adolescentes y estudiantes universitarios. A raíz de estos datos, en 1937 se publicó la Marihuana Tax Act, que prohibía el consumo de cannabis, y pasaba su control a manos federales. En 1961, el cannabis se incluyó en la lista de sustancias psicotrópicas prohibidas de la Convención sobre Drogas Narcóticas y en la Lista de Estupefacientes sometidos a Fiscalización Internacional de la Convención sobre Sustancias Psicotrópicas de 1971. Pese a todo ello, la marihuana se continuó consumiendo de forma clandestina, pero limitada, hasta la gran eclosión de la década de 1960, coincidiendo con el auge del movimiento hippie. A partir de ese momento, se ha producido un crecimiento progresivo de su consumo, principalmente entre la población juvenil, a ambos lados del Atlántico27.

Actualmente, el cannabis es la droga ilegal más consumida en Europa; más de 12,5 millones de europeos son consumidores (al menos una vez al mes) y más de 3 millones lo consumen a diario o casi a diario ${ }^{20}$. En España, el consumo de cannabis durante la última década ha ido creciendo progresivamente (Tabla 2). En la actualidad, la prevalencia del consumo de cannabis en el último mes es del 7,2\% de la población ${ }^{12}$, cifra que asciende al $20,1 \%$ en el rango de edad de 14 a 18 años ${ }^{28}$.

\section{LSD}

Muchos pueblos de la antigüedad utilizaban los extractos alucinógenos del hongo Claviceps purpurea (cornezuelo del centeno) para sus ritos mágicos y religiosos, como el culto a Mitra, por parte de los celtas $^{29}$. En una tablilla asiria del año 600 a.C. se advierte sobre el peligro del uso de centeno contaminado con "pústulas negras". La intoxicación causada por su consumo, el ergotismo, provocó terribles epidemias desde el año 857 hasta la actualidad. Se trataba del denominado ignis sacer ("fuego sagrado") o fuego de San Antonio. La primera noticia fehaciente que se tiene sobre los efectos del cornezuelo, en forma de epidemia, está fechada en el año 1039, en la ciudad francesa de Dauphiné, donde está enterrado San Antonio, famoso por sus visiones demoníacas. La causa de tales epidemias -pan contaminado con el hongo Claviceps purpurea- se descubrió en el siglo XVII, y a partir de entonces los brotes de envenenamiento por el cornezuelo del centeno han sido sólo esporádicos. 
En 1938, Albert Hofmann, en el curso de sus investigaciones sobre las propiedades curativas del cornezuelo del centeno en los laboratorios de la compañía Sandoz, en Basilea, añadió un grupo dietilamida al ácido lisérgico, logrando la dietilamida correspondiente, conocida como LSD, obteniéndose así uno de los alucinógenos más potentes. Cinco años más tarde, en 1943, se descubrió, por azar, la potencia y los efectos psicotomiméticos de este fármaco $^{30}$. Trabajando con la sustancia número 25 derivada del LSD (LSD-25), Hofmann se intoxicó y comenzó a sentirse mareado y a tener visiones extrañas, debiendo refugiarse en su domicilio, en pleno delirio, hasta que cedieron los efectos de la inhalación accidental del que sería el más poderoso alucinógeno jamás conocido. De esta forma comenzó lo que sería denominada la "era psicodélica". La compañía farmacéutica suiza Sandoz comercializó el LSD, con el nombre Delysid ${ }^{\circledR}$, como una herramienta de apoyo a la terapia psicoanalítica ${ }^{31}$. El uso de esta sustancia con fines lúdicos se extendió rápidamente durante las décadas de 1960 y 1970, aprovechando los mensajes contraculturales del movimiento hippie y de las corrientes antipsiquiátricas. Toda una serie de ensayistas e intelectuales utilizaron esta sustancia en la búsqueda de distintas sensaciones, como Timothy Leary, Richard Alpert o William S. Burrough, asociando su uso a la ola contracultural de la época ${ }^{30}$. Conforme los efectos tóxicos de esta droga se fueron conociendo, su empleo clínico fue desapareciendo, aunque en la década de 1990, con la eclosión de las drogas sintéticas, el LSD ha vuelto a resurgir con fuerza, acaparando un porcentaje de mercado ilegal preocupante. En la actualidad, el consumo y el tráfico de LSD se consideran marginales, aunque las incautaciones en Europa, que han ido en aumento desde 2003, posiblemente sugieran una revitalización del interés por esta droga en los últimos años ${ }^{13}$.

\section{Anfetaminas}

Aunque la anfetamina ya había sido sintetizada en 1887 por el químico japonés Nagayoshi Nagai, las primeras evaluaciones biológicas de su actividad, enfocadas al estudio concreto de su actividad simpaticomimética, no habían determinado mayores ventajas sobre las sustancias de referencia. Este hecho paralizó el desarrollo del compuesto, hasta que las circunstancias reactivaron el mercado para las aminas simpaticomiméticas, a finales de la década de 1920. La monopolización del comercio de la Ephedra vulgaris, planta a partir de la cual se obtenía la efedrina, determinó que este principio activo escaseara y se elevase de precio, lo que resultó un caldo de cultivo idóneo para el desarrollo de nuevas alternativas terapéuticas para el tratamiento del asma o la congestión de vías respiratorias. En este contexto, a principios de los años 30 se redescubrieron los efectos presores y antisecretores de la anfetamina, y los laboratorios Smith Kline \& French comercializaron una especialidad que contenía este principio activo para uso inhalatorio como descongestionante nasal, un sulfato de anfetamina en mezcla racémica sintetizado en 1931 y denominado Benzedrina ${ }^{\circledR 32}$. La evolución de esta línea llevó a purificar el isómero activo de la anfetamina (dextroanfetamina) y a la introducción de otros derivados, como la metanfetamina (obtenida en 1929 directamente a partir de efedrina, por reducción de la misma), la fenmetrazina, etc. Más tarde, se usaron anfetaminas como agentes anorexígenos y antidepresivos.

Sin embargo, el "uso viciado" de estos fármacos se inició en plena II Guerra Mundial, cuando se recurrió a estas drogas para minimizar los efectos del cansancio de los soldados y "fomentar motivaciones patrióticas". Es bien conocido, en este sentido, como los famosos pilotos suicidas japoneses eran "invitados" a consumir altas dosis de anfetaminas antes de lanzarse contra los barcos americanos, aunque también se usaron para aumentar el rendimiento de los trabajadores en las industrias bélicas, en la retaguar$\mathrm{dia}^{24}$. Pero los verdaderos problemas asociados al consumo de anfetaminas aparecieron en el periodo de postguerra, sobre todo en Japón, pues se dio "salida" a las elevadas reservas de anfetaminas de uso militar disponibles, lo que ocasionó un gran aumento de la delincuencia, principalmente durante la década de 1960. Después de este primer uso bélico "no terapéutico", las anfetaminas pasaron al campo del deporte, donde se emplearon "ilegalmente" para mejorar el rendimiento motriz de los deportistas. A pesar de sus problemas y del importante número de muertes súbitas, estas sustancias continuaron utilizándose hasta la implementación sistemática de los controles antidopaje.

El periodo de máximo esplendor en el uso médico de las anfetaminas fue la década de 1960, en la que se pusieron en el mercado de Europa y Estados Unidos una enorme cantidad de preparados farmacéuticos con anfetaminas. A título de ejemplo, en Gran Bretaña, durante el año 1959, alrededor del 2,5\% de las prescripciones del Servicio Nacional de Salud eran preparados que contenían anfetaminas ${ }^{33}$. La comunidad médica respondió a la introducción de las anfetaminas del mismo modo en que lo había hecho 50 años antes con la cocaína, siendo, de hecho, recomendadas para las mismas indicaciones; el tratamiento de la epilepsia, esquizofrenia, depresión, sobredosis de barbitúricos, dependencia de estimulantes, 
como el café y el tabaco, obesidad, colon irritable, esclerosis múltiple, traumatismos cerebrales y disfunciones sexuales ${ }^{34}$. Sin embargo, efectos secundarios importantes, como la cardiotoxicidad, la capacidad de producir psicosis o el riesgo de producir dependencia, acabaron, a mediados de esta década, con algunas indicaciones que habían conseguido mantener una presencia comercial importante de los derivados anfetamínicos, principalmente el tratamiento de la obesidad. De esta forma, en países como Estados Unidos, la metanfetamina se retiró del mercado en 1968. No obstante, las anfetaminas continuaron siendo empleadas por las amas de casa, como instrumentos de adelgazamiento, o por camioneros y transportistas, sobre todo en Estados Unidos, que eran primados por llegar cuanto antes a sus destinos, máxime cuando transportaban mercancías perecederas, lo que hizo aumentar la incidencia de accidentes mortales, pues cuando desaparecían los efectos de estas drogas, los camioneros se dormían al volante. Finalmente, el consumo de anfetaminas se fue extendiendo hasta la población estudiantil, que las empleaban, sobre todo en tiempos de exámenes, para aumentar el tiempo de estudio, y más recientemente para prolongar el tiempo de "marcha" durante los fines de semana.

Aunque se trata de una droga de abuso con una prevalencia de consumo inferior a otras drogas, en algunas partes de Europa el consumo de anfetaminas constituye una parte importante del problema de las drogodependencias, estimándose que alrededor de 11 millones de europeos han consumido anfetaminas (2 millones durante el último año) ${ }^{13}$. En España, las cifras obtenidas varían entre el 3,8\% (consumo alguna vez en la vida) y el $0,3 \%$ (consumo en el último mes) ${ }^{12}$.

\section{Éxtasis y drogas de diseño}

Las "drogas de síntesis" o "drogas de diseño" han adquirido una enorme relevancia en las últimas décadas, habiéndose incrementado su consumo de forma casi epidémica entre ciertos grupos de jóvenes (básicamente en medios urbanos y bajo un patrón de uso de fin de semana). El prototipo de "droga de diseño", la metilendioximetaanfetamina (MDMA), conocida popularmente como "éxtasis" además de otros nombres (Adam, XTC, emphaty, essence, clarity, M\&M), fue sintetizada en 1914 en los laboratorios alemanes Merck como agente anorexígeno (número de patente 274.350), aunque no llegó a ser comercializada. Sin embargo, fue utilizada con fines de investigación por el ejército norteamericano (agente de experimentación n 1478 del Departamento Edgewood Chemical Warfare Service de la
Armada) durante las décadas de 1950 y 1960, y, durante la década de 1970, como agente facilitador de la comunicación (agente entactógeno) entre el psicoterapeuta y el paciente ${ }^{35}$. Entre otros derivados anfetamínicos de síntesis se encuentra la MDA (3,4-metilendioxianfetamina), conocida como "droga del amor". Fue sintetizada en la primera década del siglo XX y estudiada farmacológicamente con fines comerciales como inhibidor del apetito durante las décadas de 1950 y 1960, iniciándose su empleo como droga de abuso a partir de 1967. En la actualidad, existen, además de la MDMA y la MDA, medio centenar de sustancias sintetizadas con tal fin, como la 3,4-metilendioxietanfetamina (MDEA) o "Eva", la 4-metil-2,5-dimetoxianfetamina (DOM) o "STP", etc.

El desvío de la MDMA al mercado ilícito tuvo lugar en Estados Unidos a mediados de la década de 1980. De hecho, en ciudades como Dallas empezó a reemplazar a la cocaína como droga favorita de los "yuppies" y de consumidores de drogas de fin de semana. Ya a finales de dicha década desembarcó en Europa, de la mano del movimiento "Acid House", alcanzando un gran éxito entre la juventud, dada la facilidad de su administración, así como su bajo precio. Este movimiento encontró su máxima expresión en la isla de Ibiza. Posteriormente se exportó a la Península, siendo un elemento clave de la denominada "Ruta del Bakalao" que, bajo la bandera de la "música máquina", gozó de gran predicamento entre ciertos grupos de jóvenes durante los años 19931996, y se asoció a una gran accidentalidad en los fines de semana ${ }^{35}$.

La MDMA era ya una de las sustancias más decomisadas a mediados de la década de 1980 en Estados Unidos, y en 1985 fue incluida en la Lista I del Convenio sobre Sustancias Psicotrópicas de la Comisión de Estupefacientes de la ONU, lo que significa que está prohibida, a nivel internacional, su fabricación y venta, así como la importación, tránsito, comercio y tenencia ${ }^{35}$. En España quedó incluida en su correspondiente Lista, por Orden Ministerial de 6 de junio de 1986. Aún así, en la actualidad, entre el 0,1\% y el 3,7\% de los jóvenes europeos (15-34 años) ha consumido esta droga durante el último año ${ }^{20}$. En España, el éxtasis es la tercera sustancia ilegal por orden de prevalencia de consumo $(1,1 \%$ de la población general y $2,4 \%$ de la población adolescente en los 12 meses previos), caracterizándose este consumo, al igual que la cocaína, por su naturaleza esporádica ${ }^{12,28}$.

En los últimos años se ha incrementado el tráfico y consumo de otras drogas de diseño, procedentes de los arsenales terapéuticos "legales", como el 
gamma-hidroxibutirato (GHB), la ketamina, la fenciclidina o el JWH-018. El GHB fue sintetizado por Henri Laborit hace aproximadamente 30 años, y es una sustancia depresora del sistema nervioso central que se conoce vulgarmente con el nombre de "éxtasis líquido". Esta denominación es bastante errónea y suele conducir a peligrosas equivocaciones en los consumidores, ya que al ser un agente depresor, ocasiona efectos diametralmente opuestos a los del auténtico "éxtasis", que es una droga de carácter psicoestimulante. Durante los años 80, el GHB fue comercializado de forma libre por la industria alimenticia como estimulante de la hormona del crecimiento, utilizándose habitualmente en los gimnasios, aunque también formó parte de la composición de algunos medicamentos OTC que se dispensaban como sedantes. En relación con su prescripción ética, se ha utilizado fundamentalmente como anestésico general. En Estados Unidos se prohibió su venta, en 1990, como medicamento para el tratamiento del insomnio y la narcolepsia, tras haberse notificado 57 casos de reacciones adversas, que iban desde náuseas y vómitos, pasando por trastornos respiratorios, hasta convulsiones y estados de coma. A partir de ese momento sólo se permitió su uso bajo prescripción y supervisión médica ${ }^{36}$, hasta que, en el año 2002, el GHB fue incluido en la lista de sustancias fiscalizadas internacionalmente, siendo prohibida tanto su venta, como su consumo.

La ketamina es un anestésico general disociativo, no barbitúrico y no narcótico. Su uso terapéutico habitual es como anestésico general, de potencia levemoderada, por lo que se ha venido empleado especialmente en niños y ancianos. Sus características psicodélicas fueron descubiertas casualmente, después de que algunos pacientes, tras recuperarse de la anestesia, informaran de estos efectos ${ }^{37,38}$. Estudios específicos posteriores confirmaron que dosis inferiores a las anestésicas se asociaban a una experiencia psicodélica de gran intensidad ${ }^{37}$. Durante los últimos años, el desvío de la ketamina ("ketas", "special K") hacia el mercado ilegal, para su uso recreativo, ha ido en continuo aumento, junto a las denominadas "drogas de síntesis". Esta sustancia es apreciada por los consumidores por su capacidad de combinar efectos hipnóticos, analgésicos y amnésicos, sin pérdida de consciencia ni depresión respiratoria $^{39}$. La fenciclidina (PCP) ${ }^{40,41}$ es otro anestésico disociativo, introducido en el mercado en la década de 1950 y retirado del mismo precisamente a consecuencia de los episodios excitatorios y las alucinaciones que se observaban durante la recuperación de la anestesia, lo que no evitó su derivación al consumo ilegal ("polvo de ángel") ${ }^{1}$. El consumo de fenciclidina puede desencadenar fácilmente un cuadro psicótico (se puede observar incluso tras una sola dosis), provoca graves alteraciones comportamentales, está a menudo asociado a episodios de violencia, parece más adictógeno que el de otros psicoestimulantes y puede conducir mucho más fácilmente a una reacción fatal ${ }^{40}$. En los últimos años, nuevas sustancias de síntesis, desarrolladas inicialmente por la industria farmacéutica, se están incorporando al mercado ilegal de las drogas de abuso, que no para de crecer. Un ejemplo ilustrativo es el JWH-018 (1pentil-3-(1-naftoil)indol), un análogo sintético del $\Delta^{9}$. tetrahidrocannabinol (THC) sintetizado por el químico orgánico de la Clemson University John W. Huffman y que actúa como agonista total de los receptores cannabinoides. Esta sustancia, conocida como "Spice", "K2" o "cannabis sintético" fue introducida en los mercados europeos en 2004. A pesar de que las agencias responsables de los diferentes países están alertando del incremento del consumo de todas estas nuevas drogas de diseño, aún no hay datos fidedignos de prevalencia, debido en parte a su reciente introducción en los mercados ilegales y a la confusión de los consumidores a la hora de cumplimentar las encuestas epidemiológicas.

\section{Bibliografía}

1. Escohotado A. Historia General de las Drogas. Madrid: Alianza Editorial 2004.

2. Alamo C, López-Muñoz F. Historia de los fármacos analgésicos (I): Agentes opioides. Rev Soc Esp Dolor. 2006;13(Supl. 1):13-33.

3. González E, López-Muñoz F, Guerra JA, Antequera R. Agentes psicotrópicos y criminología: imito histórico o realidad?. En: López-Muñoz F, Alamo C. Historia de la Psicofarmacología, Tomo III, La consolidación de la psicofarmacología como disciplina científica: aspectos ético-legales y perspectivas de futuro. Madrid: Editorial Médica Panamericana, S.A. 2007;1359-401.

4. Chang HP. Commissioner Lin and the Opium War. Cambridge: Harvard University Press 1964.

5. Fay PW. The Opium War, 1840-1842. New York: W. W. Norton \& Company 1975. 
6. Waley A. The Opium War Through Chinese Eyes. Stanford: Stanford University Press 1968.

7. Macht DI. The history of opium and some of its preparation and alkaloids. JAMA. 1915;64:477-81.

8. Schmitz R. Friedrich Wilhelm Sertürner and the discovery of morphine. Pharm Hist. 1985;27:61-74.

9. Latimer D, Goldberg J. Flowers in the blood. New York: Franklin Watts 1981.

10. López-Muñoz F, Alamo C. La síntesis de la morfina: del milagro de la analgesia a la maldición de las dependencias. Redes Invest Medicament. 2007;9:15-7.

11. Rubio G, López-Trabada JR, Pascual J, Santo-Domingo J. Utilización de las drogas de abuso en el tratamiento de las enfermedades mentales. En: López-Muñoz F, Alamo C. Historia de la Neuropsicofarmacología. Una nueva aportación a la terapéutica farmacológica de los trastornos del Sistema Nervioso Central. Madrid: Ediciones Eurobook S.L. y Servicio de Publicaciones de la Universidad de Alcalá 1998;325-34.

12. Observatorio Español sobre Drogas (OED). Informe de la Encuesta Domiciliaria sobre Alcohol y Drogas en España (EDADES) 2007/08. Madrid: Ministerio de Sanidad y Consumo 2008. Disponible en: http://www.pnsd.msc.es/Categoria2/observa/pdf/InformeEdades2007-2008.pdf.

13. Observatorio Europeo de las Drogas y las Toxicomanías (OEDT). Informe anual 2008: el problema de la drogodependencia en Europa. Disponible en www.emcdda. europa.eu/publications/annual-report/2008.

14. Cabrera Bonet R, Cabrera Forneiro J. Las drogas de abuso: Un reto sanitario. Madrid: Universidad Pontificia Comillas 1994.

15. Pelt JM. Drogas y plantas mágicas. París: Editorial Fayard 1983

16. Kennedy J. Coca Exotica. The illustrated history of cocaine. New York: Fairleigh Dickinson University Press and Cornwall Books 1985.

17. Karch SB. The pathology of drug abuse. Boca Raton: CRC Press 1996.

18. Grinspoon L, Bakalar JB. Cocaine: Une drogue et son evolution socials. Quebec: Lêtincelle 1978.

19. Pascual F. Aproximación histórica a la cocaína. De la coca a la cocaína. Adicciones. 2001;13:7-22.

20. Observatorio Europeo de las Drogas y las Toxicomanías (OEDT). Informe anual 2010: el problema de la drogodependencia en Europa. Luxemburgo: Oficina de Publicaciones de la Unión Europea 2010. Disponible en: http://www.emcdda.europa.eu/publications/annual-report/2010.

21. Grinspoon L, Bakalar JB. Marihuana. La medicina prohibida. Barcelona: Paidos 1997.
22. Abel EL. Marihuana: The first twelve thousand years. New York: Plenum Press 1980.

23. Reininger W. Remnants from historic times. En: Andrews G, Vinkenoog S. The Book of Grass: An Anthology on Indian Hemp. Londres: Peter Owen Limited 1967;14-5.

24. Cabrera J. Las drogas de abuso como sustancias psicotrópicas y neurotóxicas: breve historia de las drogas. En: López-Muñoz F, Alamo C. Historia de la Psicofarmacología, Tomo II, La revolución de la psicofarmacología: sobre el descubrimiento y desarroIlo de los psicofármacos. Madrid: Editorial Médica Panamericana, S.A. 2007;1059-78.

25. McKim WA. Drugs and Behaviour. New Jersey 1991.

26. Evans FJ. The medicinal chemistry of cannabis: O 'Shaugnessy's legacy. Pharm Sci. 1997:3;533-7.

27. Ramos JA, Fernández-Ruiz J. Uso de los cannabinoides a través de la historia. Adicciones. 2000;12:19-30.

28. Observatorio Español sobre Drogas (OED). Informe de la Encuesta Estatal sobre Uso de Drogas en Estudiantes de Enseñanzas Secundarias (ESTUDES). Madrid: Ministerio de Sanidad y Consumo 2007. Disponible en: http://www.pnsd.msc.es/Categoria2/observa/pdf/Estudes2006.pdf.

29. Caro PM. Drogas de Abuso. Buenos Aires: Ed. La Roca 1997.

30. Clervoy P, Rafizadeh-Kabe JD. Agentes psicotomiméticos. Descubrimiento e historia del LSD. En: López-Muñoz F, Alamo C. Historia de la Psicofarmacología, Tomo II, La revolución de la psicofarmacología: sobre el descubrimiento y desarrollo de los psicofármacos. Madrid: Editorial Médica Panamericana, S.A. 2007;1137-50.

31. Ulrico R, Patten B. The rise, decline and fall of LSD. Perspect Biol Med. 1991;34:561-78.

32. Alguacil LF, Pérez-García C, Morales L. Fármacos estimulantes del sistema nervioso central. En: López-Muñoz F, Alamo C. Historia de la Psicofarmacología, Tomo II, La revolución de la psicofarmacología: sobre el descubrimiento y desarrollo de los psicofármacos. Madrid: Editorial Médica Panamericana, S.A. 2007;927-42.

33. Connell PH. El uso y abuso de las anfetaminas. The Practitioner. 1968;32:44-51.

34. Bett W. Benzedrine sulphate in clinical medicine: A survey of the literature. Postgrad Med J. 1946;22:205.

35. López-Muñoz F, Rubio G, González-Martínez E, Alamo C. Extasis (3,4-metilendioximetanfetamina, MDMA): Aspectos farmacológicos, clínicos y criminológicos. Trast Adict. 2004;6:16-38. 
36. Nicholson KL, Balster RL. GHB: a new and novel drug of abuse. Drug Alcohol Depend. 2001;63:1-22.

37. Krystal JH, Karper LP, Seibyl JP, Freeman GK, Delaney R, Bremner JD, Heninger GR, Bowers MBJ, Charney DS. Subanesthetic effects of the noncompetitive NMDA antagonist, ketamine, in humans. Psychotomimetic, perceptual, cognitive, and neuroendocrine responses. Arch Gen Psychiatry. 1994;51:199-214.

38. Kohrs R, Durieux ME. Ketamine: teaching an old drug new tricks. Anesth Analg. 1998;87:1186-93.
39. Ferrándiz J, Mataix A. Las nuevas drogas y sus consecuencias para la salud. JANO. $2001 ; 61: 45-50$.

40. Henderson G. Phencyclidine: a widely abused but little understood psychotomimetic agent. Trends Pharmacol Sci. 1982;3:248-50.

41. National Institute on Drug Abuse. Hallucinogens and dissociative drugs. NIDA Research Report Series, 2001. Disponible en: www.drugabuse. gov/ResearchReports/Hallucinogens/Hallucinogens.html. 